\title{
Inducible Expression of Agrobacterium Virulence Gene VirE2 for Stringent Regulation of T-DNA Transfer in Plant Transient Expression Systems
}

\author{
Erna Denkovskienè, ${ }^{1,3}$ Šarūnas Paškevičius, ${ }^{1}$ Stefan Werner, ${ }^{2}$ Yuri Gleba, ${ }^{2}$ and Aušra Ražanskiene் ${ }^{1}$ \\ ${ }^{1}$ Nomads UAB, Graičiūno 8, LT-02241, Vilnius, Lithuania; ${ }^{2}$ Nomad Bioscience GmbH, Biozentrum Halle, Weinbergweg 22, \\ D-06120 Halle (Saale), Germany; ${ }^{3}$ Institute of Biotechnology of Vilnius University, Graičiūno 8, LT-02241, Vilnius, Lithuania
}

Submitted 29 June 2015. Accepted 5 July 2015.

\begin{abstract}
Agrotransfection with viral vectors is an effective solution for the transient production of valuable proteins in plants grown in contained facilities. Transfection methods suitable for field applications are desirable for the production of high-volume products and for the transient molecular reprogramming of plants. The use of genetically modified (GM) Agrobacterium strains for plant transfections faces substantial biosafety issues. The environmental biosafety of GM Agrobacterium strains could be improved by regulating their T-DNA transfer via chemically inducible expression of virE2, one of the essential Agrobacterium virulence genes. In order to identify strong and stringently regulated promoters in Agrobacterium strains, we evaluated isopropyl- $\beta$-D-thiogalactoside-inducible promoters $\mathbf{P}_{\text {lac }}, \mathbf{P}_{\text {tac }}, \mathbf{P}_{\text {T7/laco }}$, and $\mathbf{P}_{\text {T5/lacolaco }}$ and cumic acid-inducible promoters $\mathbf{P}_{\text {lac } \text { V 5/CuO }}, \mathbf{P}_{\text {tac } / \mathrm{CuO}}, \mathbf{P}_{\mathrm{T} 5 / \mathrm{CuO}}$, and $\mathbf{P}_{\text {virE } / \mathrm{CuO} \text {. Nicotiana }}$ benthamiana plants were transfected with a virE2-deficient A. tumefaciens strain containing transient expression vectors harboring inducible virE2 expression cassettes and containing a marker green fluorescent protein (GFP) gene in their T-DNA region. Evaluation of T-DNA transfer was achieved by counting GFP expression foci on plant leaves. The virE2 expression from cumic acid-induced promoters resulted in 47 to $72 \%$ of wild-type T-DNA transfer. Here, we present efficient and tightly regulated promoters for gene expression in A. tumefaciens and a novel approach to address environmental biosafety concerns in agrobiotechnology.
\end{abstract}

Transient expression systems are widely used for small-scale protein expression in plants (Xu et al. 2012). Among several strategies used for transient transformation, including Agrobacterium infiltration and virus vector infection methods, the combination of these two-agrobacterial delivery of plant virusbased expression vectors-holds particular promise. This combinatorial approach seems to be the most universal method to obtain high-level transient expression of polypeptides or heterooligomeric proteins in just days after the initial molecular cloning event (Rybicki 2010). Several very efficient approaches of agroviral transformation are currently being used, from Icon Genetics' 'deconstructed' viral vectors (Gleba et al. 2005) to

Corresponding author: A. Ražanskiene; Telephone: +1 00370679 65278; E-mail: ausra@nomadsbio.lt

*The $\boldsymbol{e}$-Xtra logo stands for "electronic extra" and indicates that two supplementary figures and one supplementary table are published online.

(c) 2015 The American Phytopathological Society launch vectors (Musiychuk et al. 2007) and combinations of transient and stable transformations that allow for in-plant activation of viral amplification based on the transgenic expression from inducible promoters (Werner et al. 2011).

Transient plant transformation is not only a very convenient method for efficient production of biomolecules in plants, it also reduces biosafety concerns related to unintended transgene spread, because the transformation of plant tissue occurs via cytoplasmic viral RNA amplification (Marillonnet et al. 2004). Despite being nonpathogenic to humans and animals, the unwelcome spread of GM Agrobacterium strains in soil or the transformation of unintended hosts must be considered with agro-mediated plant transfections. While the production of high value proteins in plants in contained facilities is economically viable and minimizes the environmental biosafety concerns of agrotransfections, field applications would be necessary to achieve the production of lower-value and high-volume products such as amylases or cellulases in industrially relevant scale (Hahn et al. 2015; Tusé et al. 2014). Additionally, field application would be essential for transient molecular reprogramming of plants to modulate agronomic traits, such as resisting stresses or altering the quantity and quality of useful plant secondary metabolites (Glenn et al. 2013; Sainsbury and Lomonossoff 2014). While some strains of GM Agrobacterium strains are already approved for open field use in the United States, such as Agrobacterium radiobacter KI026 (Gleba et al. 2014), the release of GM agrobacteria into an open field environment still requires an assessment of potential environmental biosafety risks compared with the potential benefit from the biotechnological product. Among several possible ways to reduce the environmental risks associated with the release of a GM Agrobacterium sp., a promising one is the introduction of multiple auxotrophy and genetic manipulations to prevent conjugative plasmid transfer (Gleba et al. 2014). The environmental safety of GM Agrobacterium strains would be further increased by controlling its T-DNA transfer. If T-DNA transfer could be controlled so that GM Agrobacterium strains could only transfer a target T-DNA with the application of an inducer and lose that ability with the depletion of the inducer, it would provide additional biosecurity and dramatically reduce the environmental risks associated with field use of GM Agrobacterium strains. In order for such a system to be biosafe, industrially useful, and economically relevant, it must prevent background expression in the absence of the inducer and provide high transformation efficiency upon induction. With that goal in mind, we chose the Agrobacterium virulence gene virE2 for T-DNA transfer regulation. The VirE2 protein participates in a protection of Agrobacterium T-DNA in a plant cell and is one of the essential virulence proteins that allows for T-DNA 
transfer. VirE2 mutants of A. tumefaciens lose almost all their ability to transfer T-DNA (Citovsky et al. 1992). In our search for the strongest and most stringently regulated promoters for expression of virE2 in Agrobacterium strains, we evaluated efficiency of isopropyl- $\beta$-D-thiogalactoside (IPTG)-inducible promoters $\mathrm{P}_{\text {lac }}, \mathrm{P}_{\text {tac }}, \mathrm{P}_{\text {T5/lacolaco }}, \mathrm{P}_{\text {T7/laco, }}$, and the cumic acidinducible hybrid $\mathrm{P}_{T 5 / C u O}, \mathrm{P}_{t a c / C u O}, \mathrm{P}_{l a c U V 5 / C u O}$, and $\mathrm{P}_{v i r E / C u O}$ promoters in A. tumefaciens. Cumic acid-inducible systems showed extremely low background expression of virE2 and were highly inducible in A. tumefaciens.

\section{RESULTS}

\section{The experimental setup.}

T-DNA transfer assays were carried out in N. benthamiana plants. Two types of plant virus-based binary vectors were used for T-DNA transfer assays. Both Tobacco mosaic virus (TMV)based pICH18711 (Hahn et al. 2015) and Potato virus X (PVX)based $\mathrm{pICH} 27566$ vectors contain viral polymerase and green fluorescent protein (GFP) coding sequences in their T-DNA region. Additionally, they both also contain coding sequences of proteins responsible for viral replication and movement within plant tissues. pICH18711 has a TMV movement protein, which is responsible for the intercellular spread of viral replicons through plasmodesmata, and pICH27566 contains a set of three proteins (triple gene-block proteins) and a coat protein, which, together, are responsible for intercellular and systemic replicon spread throughout all of the plant vasculature (Fig. 1). Transient expression assays in $N$. benthamiana following agroinfiltration with A. tumefaciens GV3101 transformed with either pICH18711 or pICH27566 show strong GFP fluorescence in plant leaves 4 to 5 days postinfiltration. In these assays, the number of T-DNA transfer events can be easily quantified, as it corresponds to the number of GFP expression foci.

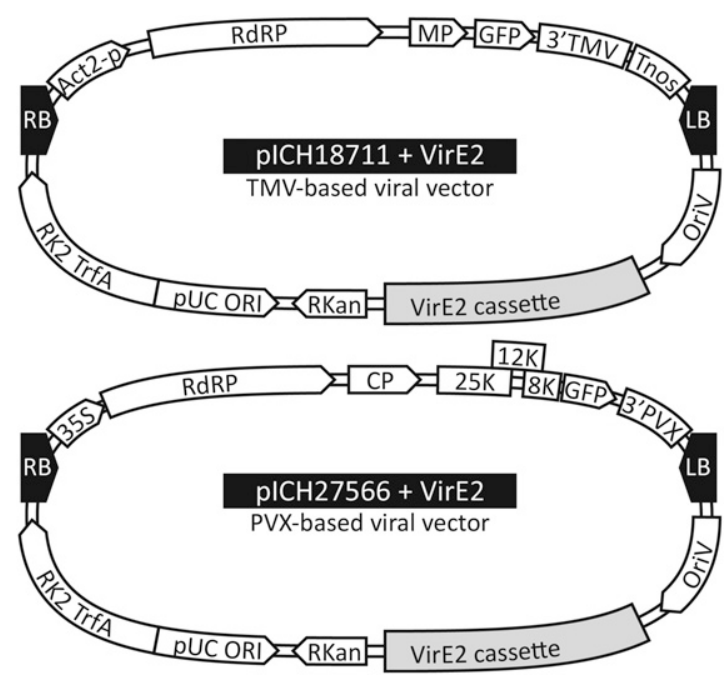

Fig. 1. Tobacco mosaic virus (TMV)- and Potato virus $X$ (PVX)-based vectors containing the virE2 expression cassette. Inducible virE2 expression cassettes were inserted into the unique Eco47III site in the backbone of either TMV-based pICH18711 or PVX-based pICH27566 and were transformed into Agrobacterium tumefaciens ICH011 (AvirE2), lacking the entire virE2 open reading frame. Transformed agrobacteria were used in agroinfiltration or agrospray assays. LB and RB = binary T-DNA left and right borders, respectively, Act $2=$ Arabidopsis thaliana actin 2 promoter, $35 \mathrm{~S}=$ Cauliflower mosaic virus promoter, Tnos $=$ nos terminator, $\mathrm{RdRp}=$ RNA-dependent RNA polymerase, $\mathrm{MP}=$ movement protein, $\mathrm{CP}=$ coat protein, $25 \mathrm{~K}, 12 \mathrm{~K}$, and $8 \mathrm{~K}=\mathrm{PVX}$ triple gene block, GFP = green fluorescent protein, $3^{\prime} \mathrm{TMV}=3^{\prime}$ untranslated region of $\mathrm{TMV}$, and $3^{\prime} \mathrm{PVX}=$ $3^{\prime}$ untranslated region of PVX.
An A. tumefaciens strain, ICH011 ( $\Delta$ virE2), lacking the entire virE2 open reading frame was constructed for inducible virE2 cassette evaluation. The transient expression assay in $N$. benthamiana plants infiltrated either with ICH011(pICH18711) or ICH011(pICH27566) showed no detectable GFP expression, thus confirming ICH011 inability to accomplish the T-DNA transfer (Fig. 2A). When transgenic $N$. benthamiana $\left(\mathrm{P}_{355}:\right.$ :virE2) plants constitutively expressing virE2 were used for agroinfiltration, both ICH011(pICH18711) and ICH011(pICH27566) were able to accomplish the T-DNA transfer, as confirmed by strong GFP fluorescence (Fig. 2A). These data are in complete agreement with the data describing the ability of VirE2 protein to complement a virE2 mutant when provided in trans (Gelvin 1998; Otten et al. 1984).

We constructed inducible virE2 cassettes and inserted them into the backbone of both pICH18711 and pICH27566, which were then transfected into strain ICH011 ( $\Delta$ virE2). The suitability of various inducible promoters to create an Agrobacteriummediated transient plant transformation system with greater environmental biosafety was assessed by comparing their ability to restore the T-DNA transfer in their induced state while exhibiting minimal background expression when not induced. All constructs were first tested in plant syringe-infiltration assays, with the number of GFP fluorescence foci evaluated visually. The best-performing constructs were then evaluated in large-scale quantitative plant spraying assays on $N$. benthamiana plants (agrospray).

\section{Evaluation of IPTG-inducible promoters in A. tumefaciens and their use for inducible T-DNA transfer.}

Lac promoter. The functionality of $\mathrm{P}_{l a c}$ promoter in A. tumefaciens has already been described (Chen and Winans 1991; Khan et al. 2008). The successful use of this promoter in Agrobacterium strains and the theoretical possibility to use an inexpensive and nontoxic product as an inducer in large-scale plant transfection (Viitanen et al. 2003) were our reasons for examining the $\mathrm{P}_{l a c}$ promoter.

$\beta$-galactosidase assays with Agrobacterium sp. strain GV3101 (pNMDV36) confirmed $\mathrm{P}_{\text {lac }}$ promoter activity in Agrobacterium strains. The detected activity amounted to 100 Miller units (MU) after $5 \mathrm{~h}$ of induction (Fig. 3A), which is in agreement with previously described values (Khan et al. 2008). We also tested $\mathrm{P}_{\text {lac }}$ promoter activity in the construct without the lacI repressor and found that the measured activity in this strain was slightly higher and amounted to approximately $400 \mathrm{MU}$ (not shown). We used native $\mathrm{P}_{\text {lac }}$ promoter conformation with conserved positions of the $\mathrm{O}_{1}$ and $\mathrm{O}_{3}$ operators and with an $\mathrm{O}_{2}$ operator lacking in the virE2 construct but present in lac $Z$ construct (the $\mathrm{O}_{2}$ operator is located in lacZ).

We examined the $\mathrm{P}_{\text {lac }}:$ virE2 construct in plant agroinfiltration assays in order to evaluate the T-DNA transfer efficiency provided by $\mathrm{P}_{l a c}$ controlled virE2 expression. However, we did not obtain GFP expression foci in syringe-infiltrated $N$. benthamiana plant leaves, indicating that no T-DNA transfer events occurred (Fig. 3A). We then tried several slightly different constructs (expression cassette in PVX- and TMV-based vectors, with or without lac $Y$ permease), but none of these constructs were able to induce detectable T-DNA transfer (not shown). We thus presumed that $\mathrm{P}_{l a c}$ promoter activity (approximately $100 \mathrm{MU}$ ) is not sufficiently strong to provide the amounts of VirE2 necessary for efficient T-DNA transfer.

Several engineered IPTG-inducible promoters $\left(\mathrm{P}_{\text {tac }}, \mathrm{P}_{\text {T5/acOlac } O}\right.$, and $\left.\mathrm{P}_{T 7 / l a c O}\right)$ are stronger than the $\mathrm{P}_{l a c}$ promoter and are used for recombinant protein production in Escherichia coli and other organisms. We decided to continue with the construction of lacZ and virE2 expression cassettes utilizing these promoters. 
$P_{\text {tac }}$ promoter. The virE2 coding sequence was inserted under control of hybrid $\mathrm{P}_{t a c}$ promoter (de Boer et al. 1983) in a TMVbased viral vector ( $\mathrm{pNMDV148)}$. This construct was tested in our agroinfiltration assay. In contrast to $\mathrm{P}_{\text {lac }}:$ virE2, the $\mathrm{P}_{\text {tac }}:$ virE2 expression cassette provided a sufficient amount of VirE2 for highly efficient T-DNA transfer to occur. Plant infiltration with ICH011(pNMDV148) resulted in intense GFP fluorescence. However, although very strong, $\mathrm{P}_{t a c}$ was poorly regulated by IPTG and gave similar GFP fluorescence in the noninduced state as when induced by IPTG. We tried to combat this leaky expression by providing an additional copy of lacIq in trans in a separate vector, but no significant improvements were obtained (not shown).

$P_{\text {T5/acOlaco }}$ promoter. Bacteriophage T5 early promoter $\mathrm{P}_{N 25}$ (hereafter called $\mathrm{P}_{T 5}$ ) has been described as a very strong promoter recognized by bacterial RNA polymerase (Rommens et al. 1983). The $\mathrm{P}_{T 5}$ hybrid promoter is used in Qiagen expression vectors and was tested in several microorganisms, including Agrobacterium species (Wang et al. 2000). A hybrid $\mathrm{P}_{T 5}$ promoter with two lac operator sequences, as in Qiagen pQE30 vector $\left(\mathrm{P}_{\text {T5/lacolaco }}\right)$, was used for virE2 and lacZ expression in Agrobacterium strains. A strong positive correlation between induction strength and concentration of IPTG was observed and no toxic effect of IPTG was noticed with concentrations as high as $10 \mathrm{mM}$ (Fig. 2B). A. tumefaciens GV3101 (pNMDV417), containing the $\mathrm{P}_{\text {T5/lacolaco }}:$ lacZ expression cassette, showed high $\beta$-galactosidase activity (approximately 1,500 MU) when induced with $2 \mathrm{mM}$ IPTG for $5 \mathrm{~h}$ and low activity (approximately $27 \mathrm{MU}$ ) in an uninduced state (Fig. 3B). The plant agroinfiltration assay with $A$. tumefaciens ICH011 (pNMDV519) containing the $\mathrm{P}_{\text {T5/lacolaco }}$ : virE2 expression cassette showed almost undetectable GFP fluorescence in the absence of the inducer and strong GFP fluorescence in its presence (Fig. 3B).

$P_{T 7 / \text { Iaco }}$ promoter. The bacteriophage T7 RNA polymerasedriven hybrid $\mathrm{P}_{T 7 / \text { lac }}$ promoter is one of the strongest and most stringent promoters used for heterologous protein expression in E. coli (Studier et al. 1990). It has also been tested in other microorganisms, including A. tumefaciens (Kang et al. 2007). We tested the $T 7$ expression system for inducible virE2 expression. In contrast to previously described $T 7$ expression systems, our $\mathrm{P}_{\text {lacUV5 }}:$ T7 polymerase expression cassette was not integrated into the bacterial genome but was cloned in a separate plasmid pNMDV163. The $\mathrm{P}_{\text {T7/acO }}:$ : virE2 expression cassette was cloned into pICH18711 giving construct pNMDV164. A. tumefaciens ICH011(pNMDV163, pNMDV164) was tested in our agroinfiltration assay and demonstrated the restored ability of T-DNA transfer (Fig. 3C). The efficiency of T-DNA transfer was significantly lower than transfer obtained with $\mathrm{P}_{\text {T5/acOlaco }}$ ::virE2, and some leakiness of the promoter was noticed in the absence of the inducer. We next tested the activity of $T 7$ polymeraseregulated $\mathrm{P}_{\text {T7лlaco }}$ promoter in a $\beta$-galactosidase assay using the A. tumefaciens strain GV3101(pNMDV163, pNMDV223). Surprisingly, almost no activity was detected in Agrobacterium strains even in the presence of IPTG, contradictory to our results with virE2. We retransformed both pNMDV163 and pNMDV223 plasmids into $E$. coli and, then measured $\beta$-galactosidase activity. The results showed high promoter activity, confirming the functionality of the system in E. coli (Fig. 3C). We cannot explain the observed discrepancy between lack of $\beta$-galactosidase activity in Agrobacterium strains and successful inducible T-DNA transfer. We can speculate that the observed difference is due to different induction times and conditions between the $\beta$-galactosidase activity test in liquid culture and the infiltration experiment in planta. In general, virE2 expression from $\mathrm{P}_{\text {T7/laco }}: \because$ virE2 resulted in markedly weaker GFP fluorescence in plant leaves in comparison with the wild-type strain, and as such, the obtained T-DNA transfer efficiency did not meet our goals and expectations.

\section{Evaluation of cumic acid-inducible promoters} in A. tumefaciens and their use for inducible T-DNA transfer.

A novel tightly regulated gene-expression system was recently developed for $E$. coli by applying the regulatory elements of the Pseudomonas putida $\mathrm{F} 1$ cym and cmt operons to control target gene expression at the transcriptional level by using $\mathrm{p}$-isopropylbenzoate (cumate) as an inducer. This system is referred to as the cumate gene switch (Choi et al. 2010). We evaluated the possibility of using cumate-inducible promoters in Agrobacterium tumefaciens.

$P_{\mathrm{T} 5 / \mathrm{CuO}}$ promoter. $\mathrm{P}_{T 5 / \mathrm{CuO}}:$ vvirE2 cassette was inserted into viral vectors pICH18711 and pICH27566, resulting in constructs pNMDV281 and pNMDV386, respectively. Unlike Choi et al. (2010), cumic acid (4-isopropylbenzoic acid) was used as an inducer instead of cumate ( $\mathrm{p}$-isopropylbenzoate) because cumic
A

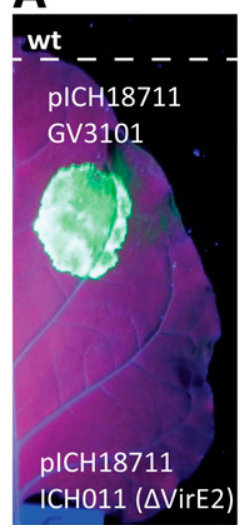

B

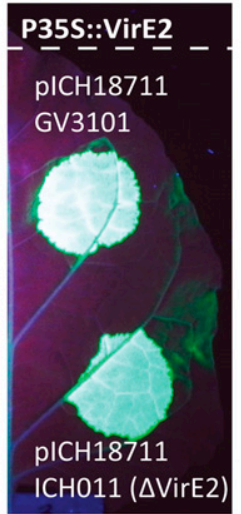

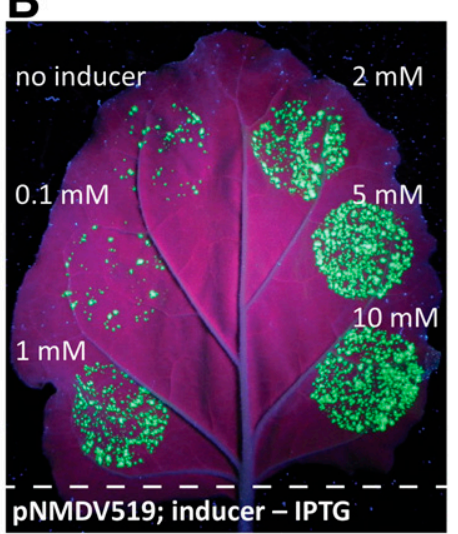

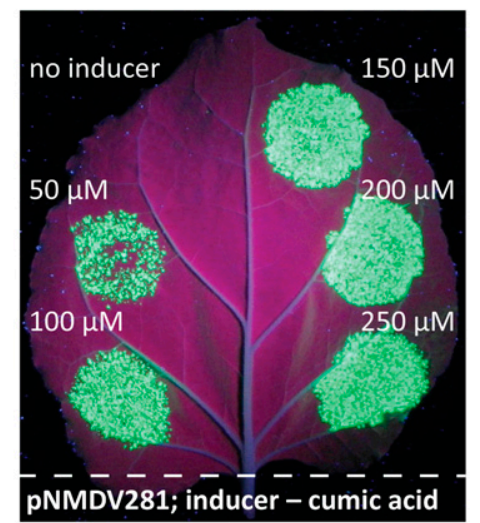

Fig. 2. T-DNA transfer by the Agrobacterium tumefaciens ICH011 strain. A, The wild-type Nicotiana benthamiana agroinfiltration with virE2 deficient A. tumefaciens ICH011 (pICH18711) shows no detectable green fluorescent protein (GFP) expression, thus confirming its inability to accomplish the T-DNA transfer. In comparison, strong GFP expression is observed with control strain GV3101(pICH18711). When transgenic $N$. benthamiana $\left(\mathrm{P}_{355}::\right.$ VirE2) plants are used for agroinfiltration, strain ICH011(pICH18711) is able to accomplish the T-DNA transfer, as confirmed by strong GFP fluorescence. B, Isopropyl- $\beta$-Dthiogalactoside (IPTG) and cumic acid-induced T-DNA transfer is regulated in a dose-responsive manner in A. tumefaciens ICH011 transformed with inducible virE2 constructs. Left panel, pNMDV519 ( $\mathrm{P}_{\text {T5/lacolac }}:$ :virE2. No inducer was added to the first sample as a negative control; $0.1,1,2,5$, or 10 mM IPTG was added to experimental samples just before infiltration. Right panel, pNMDV281 ( $\mathrm{P}_{T 5 / \text { cuo }}:$ virE2). No inducer was added to the first sample, 50, 100, 150, 200, or $250 \mu \mathrm{M}$ cumic acid was added to experimental samples just before infiltration. 
acid gave better results in Agrobacterium strains. Cumic acid induced relatively strong virE2 expression in A. tumefaciens, resulting in strong GFP fluorescence in plant leaves (Fig. 3D). In contrast to IPTG-inducible $\mathrm{P}_{\text {T5/acolaco }}$, cumic acid-inducible $\mathrm{P}_{T 5 / \mathrm{CuO}}$ did not provide any detectable GFP fluorescence in the absence of the inducer. We evaluated $\beta$-galactosidase activity of GV3101(pNMDV299) strain, harboring the $\mathrm{P}_{T 5 / \mathrm{CuO}}$ : lacZ expression cassette, and compared it with $\beta$-galactosidase activity of GV3101(pNMDV417) harboring the $\mathrm{P}_{\text {T5/acOlaco: }}:$ lacZ expression cassette (Fig. 3B). $\beta$-galactosidase activity was much higher when the $\mathrm{P}_{T 5 / \mathrm{CuO}}$ promoter was used for inducible expression of lac $Z$ and reached approximately 5,200 MU when induced, while expression from $\mathrm{P}_{\text {T5/lacOlaco }}$ promoter resulted only in approximately 1,500 MU. However, the basal activity of
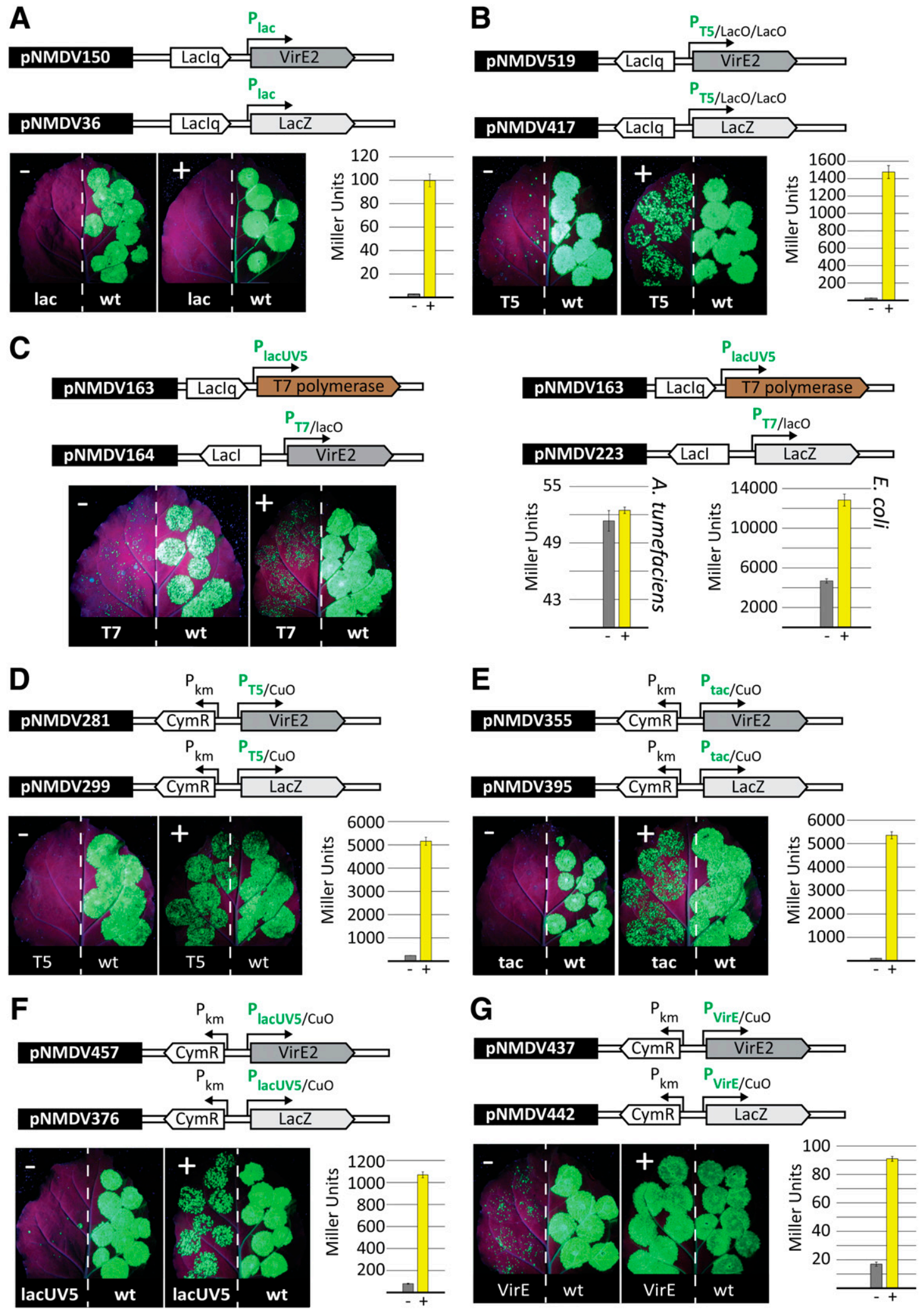
noninduced $\mathrm{P}_{T 5 / \mathrm{CuO}}$ promoter was also higher (approximately $240 \mathrm{MU}$ ) than the basal activity of $\mathrm{P}_{\text {T5/acOlac O }}$ (approximately 27 $\mathrm{MU})$. This higher leakiness of $\mathrm{P}_{T 5 / \mathrm{CuO}}$, however, did not result in detectable T-DNA transfer in the plant infiltration assays (Fig. 3D) while lower leakiness of $\mathrm{P}_{\text {T5/acolaco }}$ did (Fig. 3B). Here again, we hypothesize that expression from IPTG-inducible promoters while Agrobacterium strains are infiltrated in plant tissue may be influenced by additional factors, resulting in the observed differences with the promoter activity measured in Agrobacterium strains while in nutrient medium.

We next evaluated the correlation between cumic acid-inducible virE2 expression, resulting in T-DNA transfer and GFP fluorescence in plants, and inducer concentration. Cumic acid concentrations of 50 to $250 \mu \mathrm{M}$ were tested. As little as $50 \mu \mathrm{M}$ of cumic acid was able to induce GFP fluorescence in $N$. benthamiana leaves. This fluorescence seemed to reach its maximum induction between 100 and $250 \mu \mathrm{M}$ of cumic acid (Fig. 2B). Therefore, we chose the concentration of $150 \mu \mathrm{M}$ of cumic acid for further induction experiments.

As the next step, we analyzed the correlation between strength of expression from $\mathrm{P}_{T 5 / \mathrm{CuO}}$ and length of incubation with cumic acid. Suspensions of ICH011(pNMDV281) were incubated with $150 \mu \mathrm{M}$ cumic acid for $0.5,1,2,3,4$, and $5 \mathrm{~h}$ and, then, were syringe-infiltrated into plants. It appeared that $<0.5 \mathrm{~h}$ of incubation was sufficient for maximal induction of virE2 expression. Increased incubation time did not increase virE2 expression or transformation efficacy. Next, we determined if it would be possible to induce bacteria with cumic acid and, then, remove the inducer prior to plant infiltration. Such a procedure would have both environmental and economic benefits for large-scale plant transfection applications, because no cumic acid would be applied to the field and smaller quantities of cumic acid would be required. We induced VirE2 synthesis by cumic acid, incubated the treated bacteria for $1,2,3,4,5,6$, or $24 \mathrm{~h}$ and removed the inducer just before infiltrating bacteria into plants. Unfortunately, we did not detect any GFP fluorescence under such conditions, proving that an inducer needs to be present in the infiltration suspension to allow for efficient T-DNA transfer induction. Addition of acetosyringone to the induction medium or coexpression of VirE1, which was described as a stabilizing chaperone of VirE2 (Zhao et al. 2001), did not change the results (not shown). Therefore, it appears that the timing of VirE2 expression is crucial and that functional VirE2 cannot be preaccumulated in bacterial cytoplasm before the induction of other constituents involved in T-DNA transfer.

In summary, the first results obtained with cumic acidregulated $\mathrm{P}_{T 5 / \mathrm{CuO}}$ promoter were promising and prompted us to construct and analyze new cumic acid-regulated promoters.

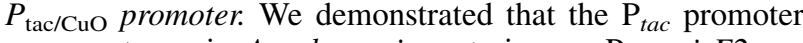
was very strong in Agrobacterium strains, as $\mathrm{P}_{\text {tac }}:$ virE2 expression resulted in very efficient T-DNA transfer. However, the $\mathrm{P}_{t a c}$ promoter was very poorly repressed in the absence of IPTG induction. Because we observed the tight regulation of $\mathrm{P}_{T 5 / \mathrm{CuO}}:$ :virE2 expression in A. tumefaciens, we expected that the $\mathrm{P}_{\text {tac }}$ promoter could also be regulated more tightly by cumic acid. A synthetic $\mathrm{P}_{t a c / C u O}$ promoter was designed by replacing the lac operator sequence in the $\mathrm{P}_{t a c}$ promoter with the cumate operator sequence. As an additional means to achieve higher stringency, the repressor gene expression cassette was cloned in opposite orientation to the $\mathrm{P}_{t a c / C u O}$ promoter, the same orientation as was utilized in the $\mathrm{P}_{T 5 / C u O}$ construct. The $\mathrm{P}_{t a c / C u O}:$ : virE2 expression cassette was inserted into viral vectors pICH18711 and pICH27566, giving constructs pNMDV355 and pNMDV356, respectively. Indeed, as anticipated, plant infiltration with the cumic acid-induced ICH011(pNMDV355) strain resulted in strong GFP fluorescence. No GFP fluorescence was observed in plant leaves infiltrated with the noninduced ICH011(pNMDV355) strain (Fig. 3E). We also tested the $\mathrm{P}_{\text {tac/CuO }}$ promoter in $\beta$-galactosidase assays, using the GV3101(pNMDV395) strain harboring the $\mathrm{P}_{\text {tac/CuO }}$ : $:$ lacZ cassette. The $\beta$-galactosidase activity was approximately $5,400 \mathrm{MU}$ in the induced state and only approximately $110 \mathrm{MU}$ in the noninduced state. These results define the hybrid $\mathrm{P}_{t a c / C u O}$ promoter as a very strong and tightly regulated promoter in Agrobacterium strains (Fig. 3E).

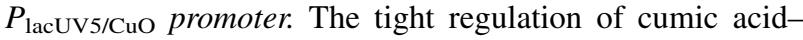
inducible promoters in Agrobacterium strains suggests that this characteristic could be exploited in order to evaluate the minimal strength of the virE2 promoter necessary for the T-DNA transfer to occur. We have previously determined that the leakiness of the $\mathrm{P}_{T 5 / \mathrm{CuO}}$ promoter (approximately $240 \mathrm{MU}$ ) is insufficient to provide enough VirE2 molecules to allow efficient T-DNA transfer to occur and that the promoter strength of $1,500 \mathrm{MU}$ of $\mathrm{P}_{\text {T5/acOlaco }}$ promoter is largely sufficient for that aim. We then searched for a promoter weaker than $\mathrm{P}_{\text {T5/acOlaco }}$. The $\mathrm{P}_{\text {lac }}$ promoter in its native conformation is weak in Agrobacterium strains and reaches only approximately $100 \mathrm{MU}$ under our induction conditions. However, we expected that by changing both the regulation of this promoter (cumic acid instead of IPTG) and the conformation of the construct (cymR gene cloned in an inverted position in comparison with lacIq in pNMDV150) we could achieve greater activity of the $\mathrm{P}_{l a c}$ promoter. We constructed a synthetic $\mathrm{P}_{\text {lac UV5/CuO }}$ promoter by replacing the lac operator sequence with a cumate operator sequence. The $\mathrm{P}_{\text {lacUV5/CuO }}:$ virE2 and $\mathrm{P}_{\text {lacUv5/CuO }}:$ lacZ expression cassettes were used to construct plasmids pNMDV457 and pNMDV376, respectively. The GV3101(pNMDV376) strain was

Fig. 3. Evaluation of inducible promoters in Agrobacterium tumefaciens ICH011. Isopropyl- $\beta$-D-thiogalactoside (IPTG) or cumic acid-inducible promoters were evaluated in $\beta$-galactosidase assays (lacZ-carrying constructs) and in plant agroinfiltration assays (virE2-carrying constructs) for their ability to restore the T-DNA transfer. A, $\mathrm{P}_{\text {lac }}$ : virE2 expression from IPTG-induced promoter did not result in detectable T-DNA transfer; the $\beta$-galactosidase activity assay showed activity of approximately 100 Miller units (MU) in Agrobacterium strains. B, $\mathrm{P}_{\text {T5/lacolaco }}$ : virE2 expression from this promoter resulted in efficient and tightly regulated T-DNA transfer; lacZ expression resulted in medium $\beta$-galactosidase activity (approximately 1,400 MU) in the induced state and very low activity (approximately $27 \mathrm{MU}$ ) without induction. $\mathbf{C}, \mathrm{P}_{T 7 / l a c} O$ : virE2 expression from this promoter resulted in moderate T-DNA transfer in the induced state and detectable T-DNA transfer in the absence of inducer. Very low $\beta$-galactosidase activity was measured in Agrobacterium when lacZ was expressed from the $\mathrm{P}_{T 7 / \text { laco }}$ promoter, while activity was high in Escherichia coli. $\mathbf{D}, \mathrm{P}_{T 5 / C u O}$ : virE2 expression resulted in highly inducible and very stringent T-DNA transfer; lacZ expression resulted in high $\beta$-galactosidase activity while induced (approximately 5,200 MU) and low leaky activity (approximately $240 \mathrm{MU}$ ). E, $\mathrm{P}_{\text {tac/CuO }}$ : virE2 expression resulted in highly inducible and very stringent T-DNA transfer; lacZ expression resulted in high $\beta$-galactosidase activity while induced (approximately $5400 \mathrm{MU}$ ) and low leaky activity (approximately $110 \mathrm{MU}$ ). F, $\mathrm{P}_{\text {lac } U V 5 / C u O}$ :virE2 expression resulted in moderately efficient and relatively stringent T-DNA transfer; $\beta$-galactosidase activity assay showed activity of approximately 1,100 MU when induced and approximately 80 MU in the absence of the inducer. $\mathbf{G}, \mathrm{P}_{\mathrm{VirE} / \mathrm{CuO}}$ : virE2 expression from the induced promoter resulted in highly efficient T-DNA transfer, however, several green fluorescent protein (GFP) expression foci were detected in the absence of the inducer; lacZ expression from this promoter resulted in low $\beta$-galactosidase activity (approximately $90 \mathrm{MU}$ ) only when $100 \mu \mathrm{M}$ acetosyringone was added to the Agrobacterium suspension. Nicotiana benthamiana leaf pictures were taken under UV light 5 days postinoculation. + indicates induction with $1 \mathrm{mM}$ IPTG or with $150 \mu \mathrm{M}$ cumic acid, - indicates no inducer added, wt = control syringe-infiltrations of Agrobacterium sp. strain GV3101 carrying viral vector with GFP. $\beta$-galactosidase assay values correspond to the average of three experiments. Bars represent standard deviation. 
A Direct and comparative evaluation of induced T-DNA transfer by agrospray (Agrobacterium suspension $\mathrm{OD}=1 \times 10^{-4}$ )

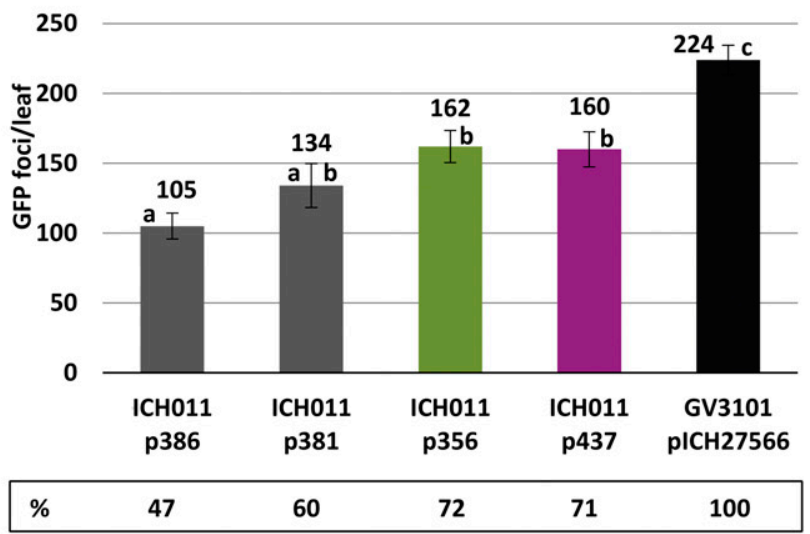

B Direct and comparative evaluation of non-induced T-DNA transfer by agrospray (Agrobacterium suspension $\mathrm{OD}=1 \times 10^{-1}$ )
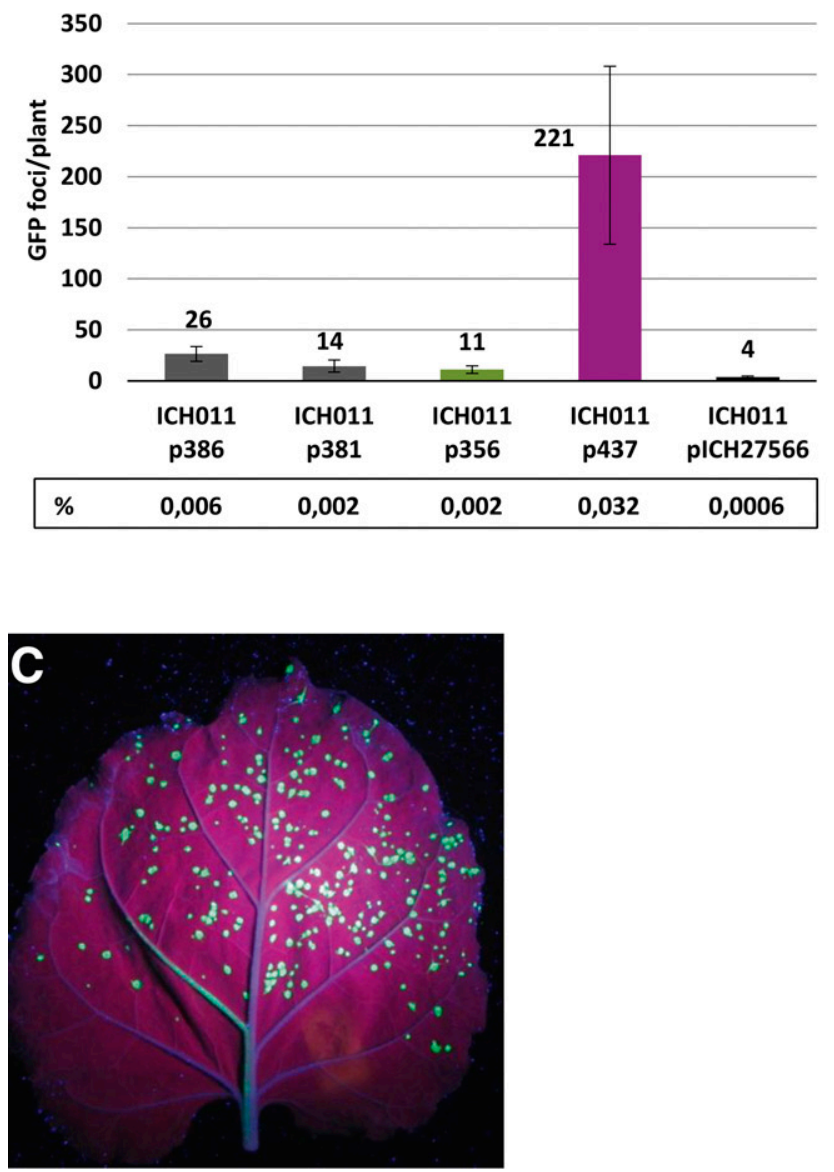

Fig. 4. Evaluation of T-DNA transfer efficiency by agrospray in A, cumic acid-induced and $\mathbf{B}$, noninduced constructs. Nicotiana benthamiana plants were sprayed with Agrobacterium strains carrying different constructs: p386 corresponds to pNMDV386 ( $\mathrm{P}_{T 5 / \text { CuO }}:$ virE2), p381 to pNMDV381

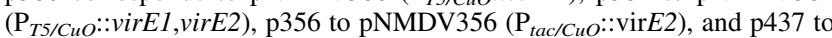
pNMDV437 ( $\mathrm{P}_{\text {VirE/CuO }:: v i r E 2)}$. The different colors indicate different promoters: gray $=\mathrm{P}_{T 5 / \mathrm{CuO}}$, green $=\mathrm{P}_{\text {tac/CUO }}$, purple $=\mathrm{P}_{\text {VirE/CuO }}$. Black columns indicate control strains. The efficiency and leakiness of T-DNA transfer in each separate experiment was evaluated as the percentage of this number in comparison with a wild-type T-DNA transfer (green fluorescent protein [GFP] expression foci number of GV3101-sprayed plants). Wildtype T-DNA transfer was considered as $100 \%$. Bars represent standard error. C, Representative picture of $N$. benthamiana leaf with GFP expression foci. tested in $\beta$-galactosidase assays and had activity of approximately $1,100 \mathrm{MU}$ when induced with cumic acid, which is considerably higher than activity of native $P_{\text {lac }}$ promoter (approximately 100 MU). The transient expression assays with the cumic acidinduced ICH011(pNMDV457) strain showed efficient T-DNA transfer in infiltrated $N$. benthamiana leaves (Fig. 3F). Only several GFP expression foci were detected when the noninduced ICH011(pNMDV457) strain was infiltrated into plant leaves (Fig. $3 F)$. The result suggests that promoter activity greater than approximately $240 \mathrm{MU}$ and less than or equal to approximately $1,100 \mathrm{MU}$ is necessary to provide minimal quantity of VirE2 protein molecules to allow efficient T-DNA transfer to occur.

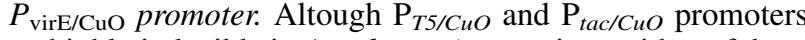
were highly inducible in Agrobacterium strains, neither of them proved itself as strong as the native virE region promoter $\mathrm{P}_{\text {virE }}$. Neither the $\mathrm{P}_{T 5 / \mathrm{CuO}}:$ virE2 nor the $\mathrm{P}_{t a c / C u O}:$ virE2 constructs could provide GFP fluorescence intensity comparable to that obtained with GV3101 strain carrying the intact native virE operon. We decided, then, to construct a cumic acid-regulated $\mathrm{P}_{\text {virE/CUO }}$ promoter. For this, we proceeded in two steps. First, the putative $\mathrm{P}_{\text {virE }}$ promoter region of Agrobacterium sp. strain KYRT-1 (Torisky et al. 1997), homologous to the described $\mathrm{P}_{\text {vire }}$ promoter in A. tumefaciens A6 (Das et al. 1986) and 99\% identical to the same region of pTiBo542 (Komari et al. 1986), was polymerase chain reaction (PCR) amplified. The cloned putative $\mathrm{P}_{\text {virE }}$ promoter was confirmed to be able to drive virE2 expression resulting in efficient T-DNA transfer (not shown). Next, we added the cumate operator sequence to the isolated $\mathrm{P}_{\text {virE }}$ promoter. The $\mathrm{P}_{\text {virE/CuO }}:$ virE2 cassette was inserted into a pICH27566 viral vector, resulting in construct pNMDV437. Plant infiltration assays with cumic acid-induced ICH011 (pNMDV437) demonstrated very strong GFP fluorescence (Fig. 3G). However, infiltration of the noninduced ICH011 (pNMDV437) strain also showed detectable GFP fluorescence, indicating greater leakiness than that of the $\mathrm{P}_{t a c / C u O}$ or $\mathrm{P}_{T 5 / C u O}$ promoters. We also constructed a $\mathrm{P}_{\text {virE/CuO }}:$ lac Z expression vector harboring Agrobacterium sp. strain GV3101(pNMDV442) and tested it in $\beta$-galactosidase activity assays. As expected, in the absence of acetosyringone, we could not detect any promoter activity in the presence or absence of cumic acid ex planta, confirming that the $\mathrm{P}_{v i r E / C u O}$ promoter needs additional factors for induction, which consist of VirG activation by plant phenolic compounds. Only when $100 \mu \mathrm{M}$ acetosyringone was used as the inducer of VirG together with cumic acid could $\beta$-galactosidase activity be detected (Fig. 3G). This activity was weak and did not correspond to the high T-DNA transfer efficiency observed in the plant infiltration assay. We presume that full induction of $\mathrm{P}_{\text {virE/CuO }}$ cannot be reached ex planta in the conditions we used.

\section{Quantitative evaluation of transfection efficiency with Agrobacterium strains carrying cumic acid-inducible virE2 constructs.}

After preliminary syringe agroinfiltrations, we performed plant sprayings with the most efficient constructs, in order to evaluate their efficiency in a large-scale transfection. The expression cassettes with $\mathrm{P}_{T 5 / C u O}, \mathrm{P}_{t a c / C u O}$, and $\mathrm{P}_{V i r E / C u O}$ promoters in PVX-based vectors in the ICH011 strain were used for these experiments. The level of wild-type T-DNA transfer obtained by spraying plants with the GV3101(pICH27566) positive control was defined as $100 \%$ efficient.

The $\mathrm{P}_{T 5 / \mathrm{CuO}}:$ :virE2 expression cassette containing ICH011 (pNMDV386) provided $47 \%$ of wild-type transfection efficiency. $\mathrm{P}_{t a c / C u O}$ and $\mathrm{P}_{V i r E / C u O}$ promoters containing strains ICH011(pNMDV356) and ICH011(pNMDV437) performed better, with T-DNA transfer efficiency reaching 71 to $72 \%$ of the wild-type expression (Fig. 4A). In order to further improve T-DNA transfer efficiency, we tried to coexpress virE1 together 
with virE2. VirE1 protein is described as a chaperone preventing VirE2 from aggregation, important for stability of VirE2, or as being helpful for VirE2 translation (Deng et al. 1999; Sundberg and Ream 1999; Zhao et al. 2001). VirE1 and virE2 coding sequences as in the native virE operon were inserted under control of the $\mathrm{P}_{T 5 / \mathrm{CuO}}$ promoter in viral vector, giving construct pNMDV381. We found that virE1 and virE2 coexpression did not significantly improve T-DNA transfer efficiency in ICH011 (pNMDV381) in comparison with virE2-expressing strain ICH011 (pNMDV386) (Fig. 4A) (analysis of variance test, $P>0.05$ ).

\section{Quantitative evaluation of transfection leakiness with Agrobacterium strains carrying cumic acid-inducible virE2 constructs.}

The leakiness from the inducible promoter cannot be avoided completely with any known inducible expression system. Even with our best-performing constructs, we observed several GFP expression foci on syringe-infiltrated plant leaves when cumic acid was not added to the Agrobacterium suspension. It was important to quantify the extent of this leakiness more precisely in $N$. benthamiana agrospray experiments.

We performed plant sprayings with all constructs previously evaluated for T-DNA transfer efficiency. In order to evaluate the significance of background expression, all tested Agrobacterium strains were diluted $10-, 10^{2}$-, and $10^{3}$-fold and were sprayed on $N$. benthamiana plants without the inducer. No GFP expression foci were detected after spraying with $10^{2}$ - and $10^{3}$-fold diluted agrobacteria. When agrobacteria were diluted only 10 fold, several GFP expression foci were detected with all virE2 expression constructs and also with the negative control strain ICH011(pICH27566). The incomplete loss of T-DNA transfer ability in the virE2-deficient strain has been already described (Citovsky et al. 1992). However, the frequency of noninduced T-DNA transfer events was extremely low and reached only 0.002 to $0.03 \%$ of wild-type T-DNA transfer (Fig. 4B). Cumic acid-inducible virE promoter showed the greatest leakiness, which, however, was 3,000 times lower than the wild-type T-DNA transfer. The leakiness of the other strong promoter $\mathrm{P}_{t a c / C u O}$ was 50,000 times lower in comparison with the wildtype T-DNA transfer and approached the leakiness obtained with the negative control.

\section{DISCUSSION}

With the evolution of technology surrounding the field of transient plant transformation, there is increasing need for innovations to address environmental biosafety issues, particularly those related to the use of GM Agrobacterium strains in open field environments. To address some of the environmental biosafety issues, we constructed expression vectors for controlled T-DNA transfer from A. tumefaciens to the host plant. We inserted an essential Agrobacterium virulence gene, virE2, under control of either IPTG- or cumic acid-inducible promoters and, then, transformed expression cassettes into a virE2-deficient A. tumefaciens strain. Due to the paucity of information available regarding the overexpression and expression control of foreign genes in A. tumefaciens, we tested several inducible promoters. We investigated several IPTG-inducible promoters and constructed several new hybrid cumic acid-regulated promoters for expression of virE2 in Agrobacterium strains. We observed better correlation between $\beta$-galactosidase activity assays and inducible T-DNA transfer with cumic acid-regulated promoters. The most efficient of the IPTG-regulated promoters was the $\mathrm{P}_{\text {T5/acOlacO }}$ promoter. The $\mathrm{P}_{T 5 / \text { IacOlaco }}$ promoter was strong and showed low leakiness in the absence of the inducer in $\beta$-galactosidase measurements. However, although this promoter seemed to be tightly regulated in the $\beta$-galactosidase assay, it demonstrated considerable leakiness in planta. In order to understand why the $\mathrm{P}_{T 5 / \mathrm{CuO}}$ promoter is less leaky in agroinfiltration experiments than $\mathrm{P}_{T 5 / \text { lacOlacO}}$, we measured, in Agrobacterium strains, the activity of the lacIq repressor promoter and the activity of the $\mathrm{P}_{K m}$ promoter used for $C y m R$ expression. The $\beta$-galactosidase activity of $\mathrm{P}_{\text {laclq }}:$ lacZ was approximately $100 \mathrm{MU}$, while $\mathrm{P}_{K m}:$ lacZ activity was four times greater and amounted to approximately 400 MU. We suggest that lacI expression from its native promoter in Agrobacterium strains may be insufficient for stringent regulation of the $\mathrm{P}_{\text {T5/lacOlacO}}$. We tried to replace lacIq with $\mathrm{P}_{K m}$ : $:$ lacI cassettes in our constructs but failed to obtain Agrobacterium transformants. Moreover, we cannot rule out other possibilities for the higher leakiness of the $\mathrm{P}_{T 5 / \text { /acOlaco }}$ promoter, i.e., different stabilities of repressors, different operator binding affinities, etc.

Different cumic acid-activated constructs were both tightly regulated and highly inducible in A. tumefaciens, both in assays in vitro and in plant transfection experiments. VirE2 expression from $\mathrm{P}_{T 5 / C u O}$ and our newly constructed hybrid promoters $\mathrm{P}_{t a c / C u O}$ and $\mathrm{P}_{\text {virE/CuO }}$ resulted in 47 to $72 \%$ efficient T-DNA transfer.

Other than promoter strength, several additional factors may influence the efficiency of inducible T-DNA transfer. As the inducer has to be cosprayed with Agrobacterium strains in order to obtain T-DNA transfer, the observed difference between the wild-type T-DNA transfer and induced T-DNA transfer efficiency might be caused by nonequal distribution and penetration of the inducer into the intercellular space of plant leaves. Also, the timing of induced virE2 expression may not correspond exactly to the timing of the wild-type virE2 expression and may be shifted temporally compared with the expression of other vir genes (i.e., chaperone VirE1 and VirB/D4 transport system, necessary for VirE2 translocation into the plant cell) (Vergunst et al. 2000). In the future, insertion of virE2 expression cassettes into the Agrobacterium genome could stabilize the system and simplify the use of inducible Agrobacterium strains. In such a case, a viral vector with any gene of interest could be transformed without further modifications into the engineered chemically regulated Agrobacterium strains and could be used for transient transfection of plants.

After examining promoter strength, we were interested in evaluating the leakiness of different virE2 expression systems. $\mathrm{P}_{\text {VirE/CuO }}$, the leakiest promoter, contains a vir box and is naturally inducible by the transcription factor VirG. VirG is highly expressed during the interaction of Agrobacterium strains and the plant cell. It is possible that high concentration of VirG may impact the leakiness of this promoter.

Low leakiness from promoters $\mathrm{P}_{t a c / C u O}$ and $\mathrm{P}_{T 5 / C u O}$, as determined by $\beta$-galactosidase assays, seemed to be insufficient to provide the necessary amount of VirE2 for T-DNA transfer in syringe-infiltration experiments. We observed no leaky T-DNA transfer from these promoters when agrospray assays were performed with diluted Agrobacterium suspensions (optical density at $600 \mathrm{~nm}\left[\mathrm{OD}_{600}\right]=0.001$ and 0.01$)$. Only when an agrobacterial density of $\mathrm{OD}_{600}=0.1$ (corresponding to approximately $10^{8} \mathrm{CFU} / \mathrm{ml}$ ) was used in agrospray assays were several T-DNA transfer events caused by leaky expression from $\mathrm{P}_{t a c / C u O}$ and $\mathrm{P}_{\text {T5/CuO }}$ detected. However, the efficiency of this observed unwanted T-DNA transfer was very low and would not be relevant under field conditions. The working bacterial concentration for agrospray is typically $10^{7}$ to $10^{6} \mathrm{CFU} / \mathrm{ml}$, which is one to two orders of magnitude less than the concentration used in our experiments to evaluate promoter leakiness. Since agrobacteria persist only at low concentrations in the field, the risk of secondary transfections resulting from promoter leakiness would be extremely low. As with any technological endeavor, we can never expect zero risk; but diligently working toward reducing environmental and human health risks to the lowest levels possible is a worthwhile and necessary goal for the adoption of new 
agronomic and biomanufacturing approaches. We believe that inducible T-DNA transfer in combination with other biosafety traits (multiple auxotrophy, programmable death of agrobacteria) could help meet the main expectations for environmental biosafety and increase trust in transient plant transformation technology for large-scale applications in the near future.

\section{MATERIALS AND METHODS}

Bacterial strains and growth conditions.

All Agrobacterium tumefaciens strains and plasmids used in this study are listed in Table 1. E. coli DH5 $\alpha$ was used as a recipient for all cloning procedures. Both E. coli and A. tumefaciens cells were grown in Luria Bertani (LB) medium at 37 or $30^{\circ} \mathrm{C}$, respectively. When necessary, all media were supplemented with ampicillin $(100 \mu \mathrm{g} / \mathrm{ml})$, spectinomycin $(50 \mu \mathrm{g} / \mathrm{ml})$, kanamycin, $(50 \mu \mathrm{g} / \mathrm{ml})$, or rifampicin $(25 \mu \mathrm{g} / \mathrm{ml})$. Restriction and modification enzymes from Thermo Scientific were used for all cloning steps.

\section{Construction of ICH011 ( $\triangle$ VirE2) strain.}

Sequences upstream and downstream of the virE2 coding sequence were PCR amplified from A. tumefaciens GV3101 genomic DNA (Supplementary Table 1) and were inserted into a pGEM-T vector (Life Technologies), resulting in constructs pICH19247 and pICH19252. pICH19247 and pICH19252 were digested with $N d e I-E c o R I$ and EcoRI-XhoI, respectively, and were ligated into vector pDNR1 (Clontech) digested with NdeIXhoI. The resulting plasmid, pICH19500, contains a $s a c B$ gene for counterselection on sucrose-containing medium. Construct pICH19500 was transformed into A. tumefaciens GV3101. The virE2 gene was deleted by two homologous recombination events as described earlier (Berger and Christie 1993). Deletion of virE2 was confirmed by PCR analysis. The resulting strain was named ICH011 (Table 1).

\section{Construction of $\mathbf{P}_{355}:$ VirE2 N. benthamiana plants.}

The virE2 coding sequence was PCR-amplified from A. tumefaciens GV3101 genomic DNA and was inserted as a NcoI-XbaI fragment into vector pICH5290 (Icon Genetics), resulting in construct pICH19160. Transcription of the virE2 gene is under the control of the Cauliflower mosaic virus $35 \mathrm{~S}$ promoter and the agrobacterial nos terminator. This vector also contains a BAR resistance cassette for selection of transgenic plants. Transgenic $N$. benthamiana plants expressing VirE2 were generated by Agrobacterium-mediated leaf disk transformation using a standard procedure. Insertion of the virE2 expression cassette was confirmed by PCR.

\section{Construction of expression cassettes.}

Description of plasmid constructs and construction schemes and sequences of synthetic cumic acid inducible promoters can be found in Supplementary Figures 1 and 2.

\section{$\beta$-galactosidase activity assay.}

The $\beta$-galactosidase activity of agrobacterial strains was determined with a colorimetric assay (Miller 1972). Agrobacterial strains were grown overnight from frozen stocks, were diluted with fresh medium to an $\mathrm{OD}_{600}$ of approximately 0.4 , and then, were incubated with shaking for $4 \mathrm{~h}$ at $30^{\circ} \mathrm{C}$ in LB medium with inducer $(150 \mu \mathrm{M}$ cumic acid [Sigma Aldrich] or $2 \mathrm{mM}$ IPTG [UAB Fermentas]) and without inducer (agrobacteria usually reached $\mathrm{OD}_{600}$ of approximately 0.8 ) to compare the levels of $\beta$-galactosidase expression.

Plant growth conditions and syringe-infiltration.

Wild-type $N$. benthamiana plants were grown in soil in an environment-controlled room at $25^{\circ} \mathrm{C}$ under long-day conditions of $16 \mathrm{~h}$ of white light and $8 \mathrm{~h}$ of dark. Five- to six-weekold plants were used for syringe-infiltration or spraying. Agrobacterium tumefaciens cultures were grown in LB medium overnight, were diluted with fresh medium to an $\mathrm{OD}_{600}=1$, and then, were further diluted $10^{3}$-fold in tap water containing IPTG or cumic acid. Syringe agroinfiltrations were performed on the abaxial side of the plant leaves. One syringe-infiltration used 200 to $300 \mu \mathrm{l}$ of diluted Agrobacterium suspension.

\section{Evaluation of T-DNA transfer efficiency and leakiness by agrospray.}

Agrobacterium strains carrying different constructs were grown overnight and were diluted with fresh medium to an $\mathrm{OD}_{600}=1$.

Table 1. Agrobacterium tumefaciens strains used in the study

\begin{tabular}{|c|c|c|}
\hline A. tumefaciens strains & Description & References \\
\hline GV3101 & (pMP90RK), nopaline, Rif $^{r}$ & (Koncz and Shell 1986) \\
\hline ICH011 & GV31014virE2 (pMP90RK), nopaline, Rif ${ }^{\mathrm{r}}$ & This study \\
\hline KYRT-1 & Disarmed Chry 5, Rif $^{\mathrm{r}}$ & (Torisky et al. 1997) \\
\hline GV3101(pICH18711) & Contains TMV virus-based vector with GFP & Icon Genetics \\
\hline GV3101(pICH27566) & Contains PVX virus-based vector with GFP & Icon Genetics \\
\hline GV3101(pNMDV36) & Contains pNMD803-lacIq, $\mathrm{P}_{l a c}:: l a c Z$ & This study \\
\hline ICH011(pNMDV148) & Contains pICH18711-lacIq, $\mathrm{P}_{\text {tac }}::$ virE2 & This study \\
\hline ICH011(pNMDV150) & Contains pICH18711-lacIq, $\mathrm{P}_{\text {lac }}::$ virE2 & This study \\
\hline GV3101(pNMDV163, pNMDV223) & $\begin{array}{l}\text { Contains pNDC-lacIq, } \mathrm{P}_{\text {lacUv5 }}:: T 7 p o l \text {, } \\
\text { pICH18711- lacI, } \mathrm{P}_{\text {T7/laco }}:: l a c Z\end{array}$ & This study \\
\hline ICH011(pNMDV163, pNMDV164) & $\begin{array}{l}\text { Contains pNDC-lacIq, } \mathrm{P}_{\text {lacUv5 }}:: T 7 p o l \\
\text { pICH18711-lacI, }, \mathrm{P}_{\text {T7/laco }}:: v i r E 2\end{array}$ & This study \\
\hline ICH011(pNMDV281) & Contains pICH18711-cymR, $\mathrm{P}_{T 5 / C u O}:: v i r E 2$ & This study \\
\hline GV3101(pNMDV299) & Contains pNDC-cymR, $\mathrm{P}_{T 5 / \mathrm{CuO}}::$ lacZ & This study \\
\hline ICH011(pNMDV355) & Contains pICH18711-cymR, $\mathrm{P}_{t a c / C u O}:: v i r E 2$ & This study \\
\hline ICH011(pNMDV356) & Contains pICH27566-cymR, $\mathrm{P}_{\text {tac/CuO }}:$ virE2 & This study \\
\hline GV3101(pNMDV376) & Contains pNDC-cymR, $\mathrm{P}_{\mathrm{lac} U V 5 / \mathrm{CuO}}::$ lacZ & This study \\
\hline ICH011(pNMDV381) & Contains pICH27566-cymR, $\mathrm{P}_{T 5 / C u O}:$ :virE, virE2 & This study \\
\hline ICH011(pNMDV386) & Contains pICH27566-cymR, $\mathrm{P}_{T 5 / C u O}:: v i r E 2$ & This study \\
\hline GV3101(pNMDV395) & Contains pNDC-cymR, $\mathrm{P}_{\text {tac/CuO }}:: l a c Z$ & This study \\
\hline GV3101(pNMDV417) & Contains pNDC-lacIq, $\mathrm{P}_{\text {T5/lacOlaco }}:$ lacZ & This study \\
\hline ICH011(pNMDV437) & Contains pICH27566-cymR, $\mathrm{P}_{\text {virE/CuO }: \text { virE2 }}$ & This study \\
\hline GV3101(pNMDV442) & Contains pICH27566- cymR, $\mathrm{P}_{\text {virE/CuO }}:$ lac Z & This study \\
\hline ICH011(pNMDV457) & Contains pICH27566-cymR, $\mathrm{P}_{\text {lacUV5/CuO }}:$ virE2 & This study \\
\hline ICH011(pNMDV519) & Contains pICH18711-lacIq, $\mathrm{P}_{\text {T5/lacOlaco }: \text { virE2 }}$ & This study \\
\hline
\end{tabular}


For testing transfer efficiency, suspensions were further diluted $1 \times 10^{4}$-fold in tap water containing $0.05 \%$ Silwet L-77 (Kurt Obermeier $\mathrm{GmbH}$ and $\mathrm{Co}$ ) and $150 \mu \mathrm{M}$ cumic acid and were incubated for $1 \mathrm{~h}$. For evaluation of leakiness, suspensions were diluted $1 \times 10^{1}$-fold in tap water plus $0.05 \%$ Silwet L-77. A 7- to 10-ml suspension was used for one plant spraying. Sprayings were performed on the abaxial side of the plant leaves. Five to seven independent induction experiments were conducted for each Agrobacterium strain. Each induction experiment utilized three to five plants with four to five leaves per plant being treated with Agrobacterium suspension. At 5 to 6 days after spraying, plant leaves were detached and were photographed under UV illumination. The number of GFP expression foci on plant leaves was counted to evaluate the frequency of the T-DNA transfer events. Average foci number and standard error were calculated and compared with wild-type T-DNA transfer.

\section{ACKNOWLEDGMENTS}

We thank A. Giritch and D. Tusé for helpful discussions and critically reading the manuscript and $\mathrm{C}$. $\mathrm{H}$. Stahl for providing language help.

\section{LITERATURE CITED}

Berger, B. R., and Christie, P. J. 1993. The Agrobacterium tumefaciens virB4 gene product is an essential virulence protein requiring an intact nucleoside triphosphate-binding domain. J. Bacteriol. 175:1723-1734.

Chen, C. Y., and Winans, S. C. 1991. Controlled expression of the transcriptional activator gene virG in Agrobacterium tumefaciens by using the Escherichia coli lac promoter. J. Bacteriol. 173:1139-1144.

Choi, Y. J., Morel, L., Le François, T., Bourque, D., Bourget, L., Groleau, D., Massie, B., and Míguez, C. B. 2010. Novel, versatile, and tightly regulated expression system for Escherichia coli strains. Appl. Environ. Microbiol. 76:5058-5066.

Citovsky, V., Zupan, J., Warnick, D., and Zambryski, P. 1992. Nuclear localization of Agrobacterium VirE2 protein in plant cells. Science 256: 1802-1805.

Das, A., Stachel, S., Ebert, P., Allenza, P., Montoya, A., and Nester, E. 1986. Promoters of Agrobacterium tumefaciens Ti-plasmid virulence genes. Nucleic Acids Res. 14:1355-1364.

de Boer, H. A., Comstock, L. J., and Vasser, M. 1983. The tac promoter: A functional hybrid derived from the trp and lac promoters. Proc. Natl. Acad. Sci. U.S.A. 80:21-25.

Deng, W., Chen, L., Peng, W. T., Liang, X., Sekiguchi, S., Gordon, M. P., Comai, L., and Nester, E. W. 1999. VirE1 is a specific molecular chaperone for the exported single-stranded-DNA-binding protein VirE2 in Agrobacterium. Mol. Microbiol. 31:1795-1807.

Gelvin, S. B. 1998. Agrobacterium VirE2 proteins can form a complex with T strands in the plant cytoplasm. J. Bacteriol. 180:4300-4302.

Gleba, Y., Klimyuk, V., and Marillonnet, S. 2005. Magnifection-A new platform for expressing recombinant vaccines in plants. Vaccine 23: 2042-2048.

Gleba, Y. Y., Tusé, D., and Giritch, A. 2014. Plant viral vectors for delivery by Agrobacterium. Curr. Top. Microbiol. Immunol. 375:155-192.

Glenn, W. S., Runguphan, W., and O'Connor, S. E. 2013. Recent progress in the metabolic engineering of alkaloids in plant systems. Curr. Opin. Biotechnol. 24:354-365.

Hahn, S., Giritch, A., Bartels, D., Bortesi, L., and Gleba, Y. 2015. A novel and fully scalable Agrobacterium spray-based process for manufacturing cellulases and other cost-sensitive proteins in plants. Plant Biotechnol. J. 13:708-716.

Kang, Y., Son, M. S., and Hoang, T. T. 2007. One step engineering of T7expression strains for protein production: Increasing the host-range of the T7-expression system. Protein Expr. Purif. 55:325-333.
Khan, S. R., Gaines, J., Roop, R. M., 2nd, and Farrand, S. K. 2008. Broadhost-range expression vectors with tightly regulated promoters and their use to examine the influence of TraR and TraM expression on Ti plasmid quorum sensing. Appl. Environ. Microbiol. 74:5053-5062.

Komari, T., Halperin, W., and Nester, E. W. 1986. Physical and functional map of supervirulent Agrobacterium tumefaciens tumor-inducing plasmid pTiBo542. J. Bacteriol. 166:88-94.

Koncz, C., and Shell, J. 1986. The promoter of the TL-DNA gene 5 controls the tissue-specific expression of chimeric genes carried by a novel type of Agrobacterium binary vector. Mol. Gen. Genet. 204:383-396.

Marillonnet, S., Giritch, A., Gils, M., Kandzia, R., Klimyuk, V., and Gleba, Y. 2004. In planta engineering of viral RNA replicons: Efficient assembly by recombination of DNA modules delivered by Agrobacterium. Proc. Natl. Acad. Sci. U.S.A. 101:6852-6857.

Miller, J. 1972: Pages 352-355. in: Experiments in Molecular Genetics. Cold Spring Harbor Laboratory, NY, U.S.A.

Musiychuk, K., Stephenson, N., Bi, H., Farrance, C. E., Orozovic, G., Brodelius, M., Brodelius, P., Horsey, A., Ugulava, N., Shamloul, A. M., Mett, V., Rabindran, S., Streatfield, S. J., and Yusibov, V. 2007. A launch vector for the production of vaccine antigens in plants. Influenza Other Respi. Viruses 1:19-25.

Otten, L., De Greve, H., Leemans, J., Hain, R., Hooykaas, P., and Scell, J. 1984. Restoration of virulence of vir region mutants of Agrobacterium tumefaciens strain B6S3 by coinfection with normal and mutant Agrobacterium strains. Mol. Gen. Genet. 195:159-163.

Rommens, J., MacKnight, D., Pomeroy-Cloney, L., and Jay, E. 1983. Gene expression: Chemical synthesis and molecular cloning of a bacteriophage T5 (T5P25) early promoter. Nucleic Acids Res. 11:5921-5940.

Rybicki, E. P. 2010. Plant-made vaccines for humans and animals. Plant Biotechnol. J. 8:620-637.

Sainsbury, F., and Lomonossoff, G. P. 2014. Transient expressions of synthetic biology in plants. Curr. Opin. Plant Biol. 19:1-7.

Studier, F. W., Rosenberg, A. H., Dunn, J. J., and Dubendorff, J. W. 1990. Use of T7 RNA polymerase to direct expression of cloned genes. Methods Enzymol. 185:60-89.

Sundberg, C. D., and Ream, W. 1999. The Agrobacterium tumefaciens chaperone-like protein, VirE1, interacts with VirE2 at domains required for single-stranded DNA binding and cooperative interaction. J. Bacteriol. 181:6850-6855.

Torisky, R. S., Kovacs, L., Avdiushko, S., Newman, J. D., Hunt, A. G., and Collins, G. B. 1997. Development of a binary vector system for plant transformation based on the supervirulent Agrobacterium tumefaciens strain Chry5. Plant Cell Rep. 17:102-108.

Tusé, D., Tu, T., and McDonald, K. A. 2014. Manufacturing economics of plant-made biologics: Case studies in therapeutic and industrial enzymes. BioMed Res. Int. 2014:256135.

Vergunst, A. C., Schrammeijer, B., den Dulk-Ras, A., de Vlaam, C. M., Regensburg-Tuïnk, T. J., and Hooykaas, P. J. 2000. VirB/D4-dependent protein translocation from Agrobacterium into plant cells. Science 290: 979-982.

Viitanen, M. I., Vasala, A., Neubauer, P., and Alatossava, T. 2003. Cheese whey-induced high-cell-density production of recombinant proteins in Escherichia coli. Microb. Cell Fact. 2:2.

Wang, Y., Mukhopadhyay, A., Howitz, V. R., Binns, A. N., and Lynn, D. G. 2000. Construction of an efficient expression system for Agrobacterium tumefaciens based on the coliphage T5 promoter. Gene 242:105-114.

Werner, S., Breus, O., Symonenko, Y., Marillonnet, S., and Gleba, Y. 2011. High-level recombinant protein expression in transgenic plants by using a double-inducible viral vector. Proc. Natl. Acad. Sci. U.S.A. 108: 14061-14066.

Xu, J., Dolan, M. C., Medrano, G., Cramer, C. L., and Weathers, P. J. 2012. Green factory: Plants as bioproduction platforms for recombinant proteins. Biotechnol. Adv. 30:1171-1184.

Zhao, Z., Sagulenko, E., Ding, Z., and Christie, P. J. 2001. Activities of virE1 and the VirE1 secretion chaperone in export of the multifunctional VirE2 effector via an Agrobacterium type IV secretion pathway. J. Bacteriol. 183:3855-3865. 